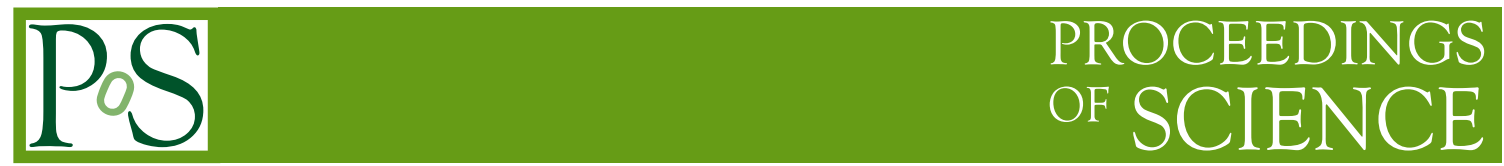

\title{
EFT Results from ATLAS
}

\section{Laura Barranco Navarro ${ }^{a, *}$ on behalf of the ATLAS Collaboration}

${ }^{a}$ The Oskar Klein Centre for Cosmoparticle Physics, Department of Physics, Stockholm University, SE-106 91 Stockholm, Sweden

E-mail: laura.barranco.navarro@cern.ch

Among all the elementary particles that constitute the Standard Model of particle physics, the top quark can provide key information on fundamental interactions at the electroweak symmetrybreaking scale and beyond. Effects of new physics can be parametrised in terms of an Effective Field Theory. This talk presents a selection of the most recent measurements provided by the ATLAS experiment from LHC accelerator in the top-quark sector that have a direct Effective Field Theory interpretation.

The Ninth Annual Conference on Large Hadron Collider Physics - LHCP2021

7-12 June 2021

Online

${ }^{*}$ Speaker 


\section{Introduction}

The Effective Field Theory (EFT) [1] provides a model-independent framework for new physics searches. Within EFT the Standard Model of particle physics (SM) is regarded as a low-energy approximation of a more fundamental theory involving interactions at an energy scale $\Lambda$. New physics is then parametrised in terms of higher-dimension operators which only include SM fields. The effective Lagrangian then becomes

$$
\mathcal{L}_{\mathrm{Eff}}=\mathcal{L}_{\mathrm{SM}}+\sum_{d, i} \frac{c_{i}^{(d)}}{\Lambda^{d-4}} O_{i}^{(d)},
$$

where $\mathcal{L}_{\mathrm{SM}}$ is the SM Lagrangian, $O_{i}^{(d)}$ are the effective operators of dimension $d$ and $c_{i}^{(d)}$ are the Wilson coefficients that parametrise the strength of the interaction. The series is usually truncated at dim-6, which provides the leading contribution of new physics.

This talk presents most recent EFT measurements from the ATLAS experiment [2-4] from LHC accelerator in the top-quark sector. All results use the Warsaw basis [5] and $\Lambda=1 \mathrm{TeV}$.

\section{Flavour Changing Neutral Currents, FCNCs}

FCNCs are forbidden at the tree level in the SM and highly suppressed at higher orders due to the GIM mechanism. However, several extensions to the SM predict processes involving FCNCs.

The analysis discussed in Ref. [6] presents the search for FCNC processes with a top quark and a photon. It uses $81 \mathrm{fb}^{-1}$ of proton-proton $(p p)$ collision data taken at a centre-of-mass energy of $13 \mathrm{TeV}$. The signal contribution is estimated from a maximum likelihood fit to the discriminant neural network output and the transverse momentum of the photon in the signal and control regions (Figure 1). From the $95 \%$ confidence level (CL) limits on the signal contribution, limits on the effective coupling parameters, production cross section and branching ratios are calculated (Table 1).

A second analysis targeting FCNCs is a search for the decay $t \rightarrow q Z$, in $36.1 \mathrm{fb}^{-1}$ of $p p$ data at $13 \mathrm{TeV}$ [7]. A $\chi^{2}$ function is built to reconstruct the signal events. The $\chi^{2}$ is fitted simultaneously with other kinematic distributions in the signal and control regions to extract the
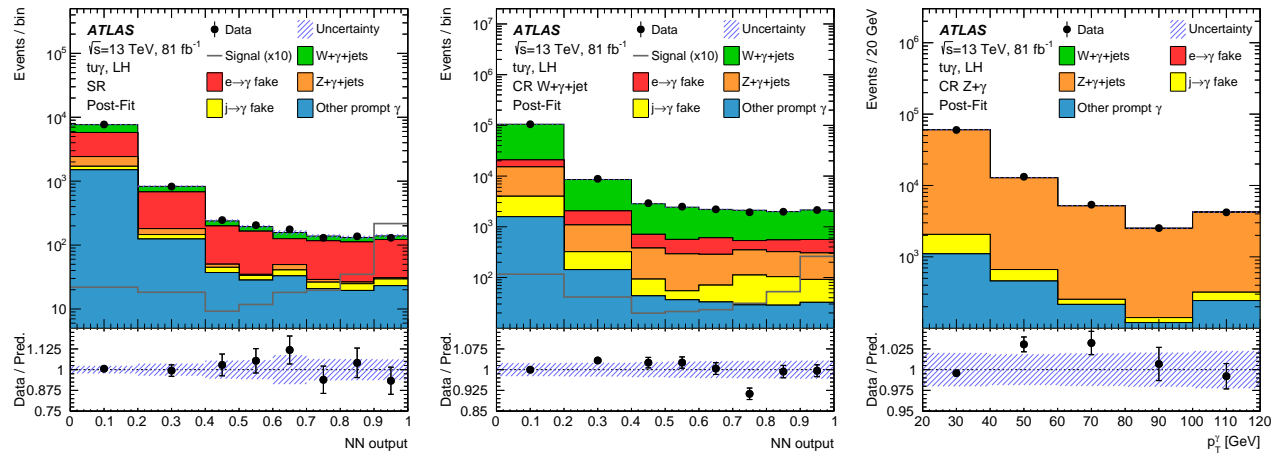

Figure 1: Post-fit distributions of a background-only fit to the Signal Region (SR) and the Control Regions (CR) of the Neural Network (NN) output in the SR (left) and the $\mathrm{W}+\gamma+$ jet CR (center) and of the $p_{\mathrm{T}^{-}}$ distribution of the $\mathrm{Z}+\gamma \mathrm{CR}$ (right) [6]. 


\begin{tabular}{ccccc}
\hline Observable & Vertex & Coupling & Obs. & Exp. \\
\hline$\left|C_{\mathrm{uW}}^{(13) *}+C_{\mathrm{uB}}^{(13) *}\right|$ & $t u \gamma$ & $\mathrm{LH}$ & 0.19 & $0.22_{-0.03}^{+0.04}$ \\
$\left|C_{\mathrm{uW}}^{(31)}+C_{\mathrm{uB}}^{(31)}\right|$ & $t u \gamma$ & $\mathrm{RH}$ & 0.27 & $0.27_{-0.04}^{+0.05}$ \\
$\left|C_{\mathrm{uW}}^{(23) *}+C_{\mathrm{uB}}^{(23) *}\right|$ & $t c \gamma$ & $\mathrm{LH}$ & 0.52 & $0.57_{-0.09}^{+0.11}$ \\
$\left|C_{\mathrm{uW}}^{(32)}+C_{\mathrm{uB}}^{(32)}\right|$ & $t c \gamma$ & $\mathrm{RH}$ & 0.48 & $0.59_{-0.09}^{+0.12}$ \\
\hline$\sigma(p p \rightarrow t \gamma)[\mathrm{fb}]$ & $t u \gamma$ & $\mathrm{LH}$ & 36 & $52_{-14}^{+2.1}$ \\
$\sigma(p p \rightarrow t \gamma)[\mathrm{fb}]$ & $t u \gamma$ & $\mathrm{RH}$ & 78 & $75_{-21}^{+311}$ \\
$\sigma(p p \rightarrow t \gamma)[\mathrm{fb}]$ & $t c \gamma$ & $\mathrm{LH}$ & 40 & $49_{-14}^{+20}$ \\
$\sigma(p p \rightarrow t \gamma)[\mathrm{fb}]$ & $t c \gamma$ & $\mathrm{RH}$ & 33 & $52_{-14}^{+22}$ \\
\hline $\mathcal{B}(t \rightarrow q \gamma)\left[10^{-5}\right]$ & $t u \gamma$ & $\mathrm{LH}$ & 2.8 & $4.0_{-1.1}^{+1.6}$ \\
$\mathcal{B}(t \rightarrow q \gamma)\left[10^{-5}\right]$ & $t u \gamma$ & $\mathrm{RH}$ & 6.1 & $5.9_{-1.6}^{+2.4}$ \\
$\mathcal{B}(t \rightarrow q \gamma)\left[10^{-5}\right]$ & $t c \gamma$ & $\mathrm{LH}$ & 22 & $27_{-7}^{+11.6}$ \\
$\mathcal{B}(t \rightarrow q \gamma)\left[10^{-5}\right]$ & $t c \gamma$ & $\mathrm{RH}$ & 18 & $28_{-8}^{+12}$ \\
\hline
\end{tabular}

Table 1: 95\% CL limits on the effective coupling strengths, production cross section, and branching ratio[6].

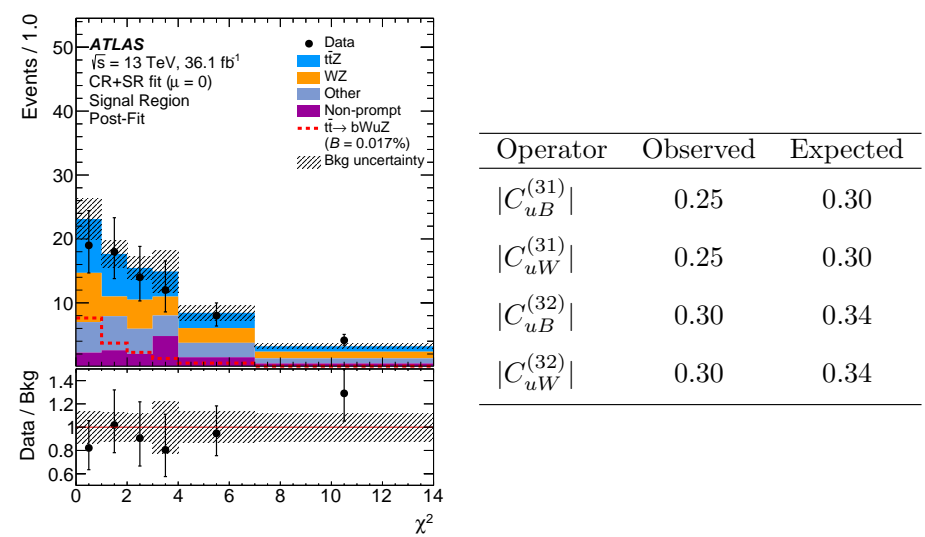

Figure 2: Post-fit distribution of a background-only fit to the SR and the CRs of the $\chi^{2}$ in the SR (left) [7]. Observed and expected 95\% CL upper limits on the moduli of the effective operators (right) [7].

signal contribution (Figure 2). From the $95 \% \mathrm{CL}$ limits on the signal contribution, limits on the effective coupling parameters are calculated (Figure 2).

\section{Charge asymmetry in top pair production}

When produced in pairs, asymmetries between the top quark and the top antiquark arise from the interference of higher-order amplitudes in the $q q$ and $q g$ initial states. Due to the difference in the parton distribution function, valence quarks carry a larger fraction of proton's momentum than the sea antiquarks, resulting in more forward top quarks and more central top antiquarks (central-forward charge asymmetry, $A_{C}$ ). $A_{C}=0.0060 \pm 0.0015$ (stat.+syst.) is measured from $139 \mathrm{fb}^{-1}$ of $p p$ data at $13 \mathrm{TeV}$ in Ref. [8] (Figure 3). Limits on a linear combination of effective couplings $\left(c_{q q}^{(8,1)}, c_{q q}^{(8,3)}, c_{u t}^{(8)}, c_{q u}^{(1)}, c_{q t}^{(1)}, c_{d t}^{(8)}, c_{q d}^{(1)}\right)$ are set from these measurements (Figure 4).

\section{Top quark polarisation}

In the $t$-channel at LO, single top quarks are produced with their spin completely aligned along the direction of the spectator quark. Since the top quark decays before it has time to hadronise, its spin information is transmitted to its decay products and the polarisation vector can be measured from angular distributions of the decay particles (in particular, that of the charged lepton and the $x$ 

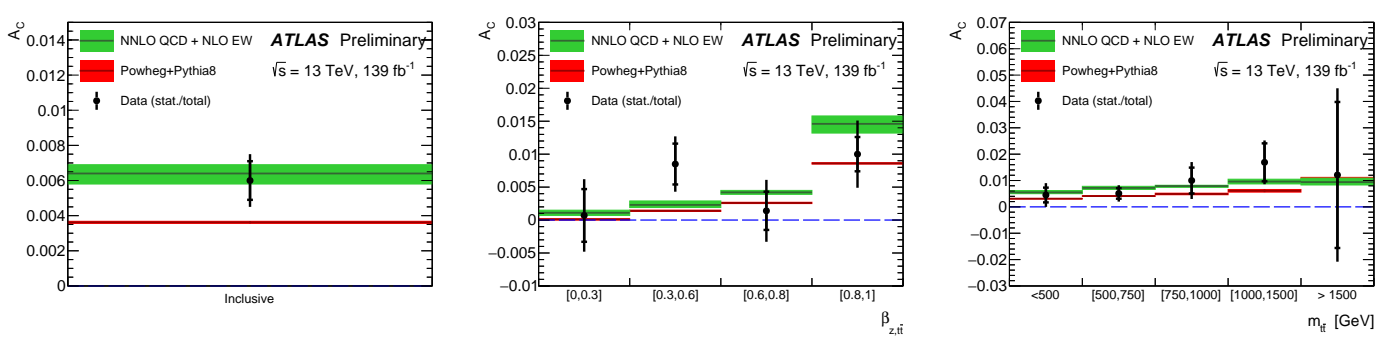

Figure 3: Unfolded inclusive (left) and differential charge asymmetries as a function of the longitudinal boost (center) and the invariant mass (right) of the top pair system in data [8].

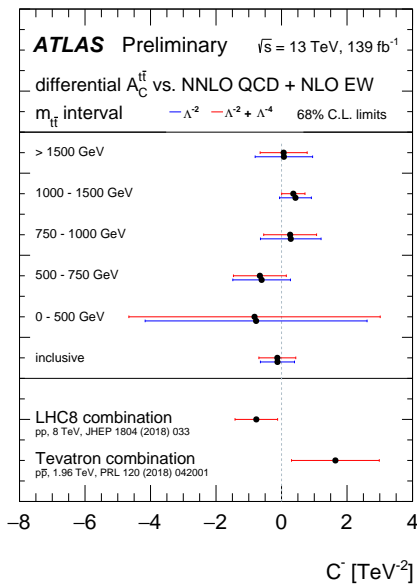

Figure 4: $68 \%$ C.L. limits on the linear combination $C^{-} / \Lambda^{2}$ of Wilson coefficients of dim-six operators [8].
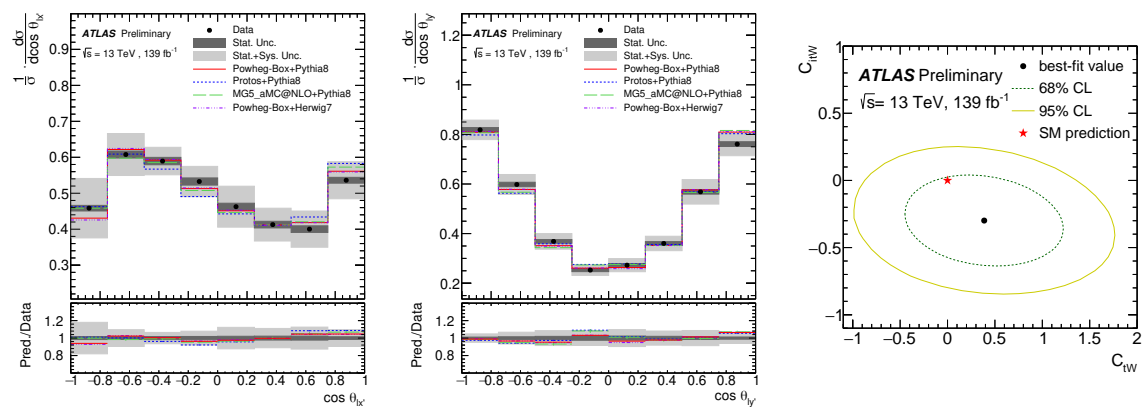

Figure 5: Particle-level differential cross-sections as a function of (left) $\cos \theta_{\ell x^{\prime}}$ and (center) $\cos \theta_{\ell y^{\prime}}$ [9]. The observed best-fit value for the Wilson coefficients $C_{t W}$ (x-axis) and $C_{i t W}$ (y-axis) (right) [9].

and $y$ directions). This is measured in Ref. [9] using $139 \mathrm{fb}^{-1}$ of $p p$ data at $13 \mathrm{TeV}$ (Figure 5). The distributions are simultaneously fitted to extract limits on the complex $t W b$ dipole operator (Figure $5)$.

\section{Summary}

The top quark provides a unique opportunity to search for new physics and EFT provides a framework to do it in a model-independent manner. This talk has shown the latest results in this context from the ATLAS experiment from the LHC accelerator at CERN. 


\section{References}

[1] W. Buchmuller and D. Wyler, Nucl. Phys. B 268 (1986), 621-653 doi:10.1016/05503213(86)90262-2

[2] ATLAS Collaboration, JINST 3 (2008), S08003 doi:10.1088/1748-0221/3/08/S08003

[3] ATLAS Collaboration, ATLAS-TDR-19, https://cds.cern.ch/record/1291633 ATLAS-TDR19-ADD-1, https://cds.cern.ch/record/1451888

[4] B. Abbott et al, JINST 13 (2018) T05008, arXiv: 1803.00844 [physics.ins-det].

[5] B. Grzadkowski, M. Iskrzynski, M. Misiak and J. Rosiek, JHEP 10 (2010), 085 doi:10.1007/JHEP10(2010)085 [arXiv:1008.4884 [hep-ph]].

[6] ATLAS Collaboration, Phys. Lett. B 800 (2020), 135082 doi:10.1016/j.physletb.2019.135082 [arXiv:1908.08461 [hep-ex]].

[7] ATLAS Collaboration, JHEP 07 (2018), 176 doi:10.1007/JHEP07(2018)176 [arXiv:1803.09923 [hep-ex]].

[8] ATLAS Collaboration, ATLAS-CONF-2019-026.

[9] ATLAS Collaboration, ATLAS-CONF-2021-027. 